\title{
Highlights of the 12th International Bordetella Symposium
}

\begin{abstract}
Camille Locht, ${ }^{1}$ Nicholas H. Carbonetti, ${ }^{2}$ James D. Cherry, ${ }^{3}$ F. Heath Damron,${ }^{4,5}$ Kathryn M. Edwards, ${ }^{6}$ Rachel Fernandez, ${ }^{7}$ Eric T. Harvill, ${ }^{8}$ Daniela Hozbor, ${ }^{9}$ Kingston H. G. Mills, ${ }^{10}$ Maria Eugenia Rodriguez, ${ }^{11}$ and Françoise Mascart ${ }^{12}$

'Université Lille, Centre National de la Recherche Scientifique, Inserm, Centre Hospitalier Universitaire Lille, Institut Pasteur de Lille, U1019, Unité Mixte de Recherche 8204, Center for Infection and Immunity of Lille, Lille, France, ${ }^{2}$ Department of Microbiology and Immunology, University of Maryland School of Medicine, Baltimore, Maryland, USA, ${ }^{3}$ David Geffen School of Medicine, University of California, Los Angeles, Los Angeles, California, USA, ${ }^{4}$ Department of Microbiology, Immunology and Cell Biology, West Virginia University, Morgantown, West Virginia, USA, ${ }^{5}$ Vaccine Development Center at West Virginia University Health Science Center, West Virginia University, Morgantown, West Virginia, USA, ${ }^{6}$ Division of Pediatric Infectious Diseases, Department of Pediatrics, Vanderbilt University Medical Center, Nashville, Tennessee, USA, ${ }^{7}$ Department of Microbiology and Immunology, Life Sciences Institute, University of British Columbia, Vancouver, British Columbia, Canada, ${ }^{8}$ Department of Infectious Diseases, College of Veterinary Sciences, University of Georgia, Athens, Georgia, USA, ${ }^{9}$ Laboratorio VacSal, Instituto de Biotecnología y Biología Molecular, Facultad de Ciencias Exactas, Universidad Nacional de La Plata, Consejo Nacional de Investigaciones Cientificas y Técnicas, La Plata, Argentina, ${ }^{10}$ School of Biochemistry and Immunology, Trinity Biomedical Sciences Institute, Trinity College Dublin, Dublin, Ireland, ${ }^{11}$ Centro de Investigacion y Desarrollo en Fermentaciones Industriales, Consejo Nacional de Investigaciones Cientificas y Técnicas, La Plata, Facultad de Ciencias Exactas, Universidad Nacional de La Plata, La Plata, Argentina, and ${ }^{12}$ Laboratory of Vaccinology and Mucosal Immunity, Université Libre de Bruxelles, Brussels, Belgium
\end{abstract}

To commemorate the 100th anniversary of the Nobel prize being awarded to Jules Bordet, the discoverer of Bordetella pertussis, the 12th International Bordetella Symposium was held from 9 to 12 April 2019 at the Université Libre de Bruxelles, where Jules Bordet studied and was Professor of Microbiology. The symposium attracted more than 300 Bordetella experts from 34 countries. They discussed the latest epidemiologic data and clinical aspects of pertussis, Bordetella biology and pathogenesis, immunology and vaccine development, and genomics and evolution. Advanced technological and methodological tools provided novel insights into the genomic diversity of Bordetella and a better understanding of pertussis disease and vaccine performance. New molecular approaches revealed previously unrecognized complexity of virulence gene regulation. Innovative insights into the immune responses to infection by Bordetella resulted in the development of new vaccine candidates. Such discoveries will aid in the design of more effective approaches to control pertussis and other Bordetella-related diseases.

Keywords. Bordetella; whooping cough; pertussis epidemiology; pertussis vaccines; Bordetella genomics.

The 1919 Nobel prize in Physiology/Medicine was awarded to Jules Bordet for his discovery of alexine, today referred to as complement. However, to the Bordetella community he is probably best known for the identification of the whooping cough agent, initially named Haemophilus pertussis and now known as Bordetella pertussis. In honor of Jules Bordet and to commemorate his Nobel Prize, the 12th International Bordetella Symposium was held from 9 to 12 April 2019, at the Université Libre de Bruxelles, where he studied and spent most of his scientific career.

\section{OPENING SESSION}

Several grandchildren and great-grandchildren of Jules Bordet were present during the opening session, and Bordet's great-granddaughter Nathalie Devroey presented a portrait of her great-grandfather with focus on his generous and warm personality. She explained how he introduced his grandchildren to scientific discovery and to

Received 12 March 2020; editorial decision 29 April 2020; accepted 22 May 2020; published online May 28, 2020.

Correspondence: C. Locht, Center for Infection and Immunity of Lille, Institut Pasteur de Lille, 1, rue du Prof. Calmette, F-59019 Lille, France (camille.locht@pasteur-lille.fr).

\section{Clinical Infectious Diseases ${ }^{\circledR} \quad$ 2020;71(9):2521-6}

(C) The Author(s) 2020. Published by Oxford University Press for the Infectious Diseases Society of America. All rights reserved. For permissions, e-mail: journals.permissions@oup.com. DOI: 10.1093/cid/ciaa651 classic literature, and how "he had the most marvelous ability to explain the most complicated things in the most understandable ways."

James D. Cherry delivered the opening lecture on the 112year odyssey of pertussis and pertussis vaccines. He highlighted the various misconceptions and their consequences for the current pertussis problem. Although on the rise in several countries, the rate of pertussis today is approximately 20 -fold less than in the prevaccine era [1]. Furthermore, vaccine failures result in less severe illness than seen in unvaccinated children. Pertussis occurs in cycles of 2-5 years, which has not significantly changed since the introduction of pertussis vaccines, indicating that vaccination does not impede B. pertussis circulation. Asymptomatic infections, a major finding in children, are 4-22 times more common than symptomatic infections. Pertussis toxin (PT) is a major virulence factor in clinical disease [2], and an as yet unidentified "cough toxin" may participate in the clinical picture of pertussis. Finally, he discussed reasons why acellular pertussis $(\mathrm{aP})$ vaccines are inferior to whole-cell $(\mathrm{wP})$ vaccines, including linked-epitope suppression, genetic changes induced by aP vaccines, a suboptimal balance of antigens, and failure to induce the appropriate T-cell subtypes. Thus, new pertussis vaccines are needed, either multicomponent aP vaccines with appropriate adjuvants, less reactogenic wP vaccines, or live attenuated vaccines. 


\section{SPECIAL SESSION ON ADENYLATE CYCLASE TOXIN}

A special session was organized as a tribute to the lifetime achievements of Erik Hewlett, who made seminal contributions to understanding the role of adenylate cyclase toxin (ACT) and played a key role in the initiation and organization of many of the previous International Bordetella Symposia and in fostering the constitution of the International Bordetella Society.

F. Heath Damron summarized Hewlett's career, from his college training in Fulton, Missouri in 1968, to his full professorship at the University of Virginia. He also highlighted Hewlett's scientific contributions to the understanding of the ACT biology and the enhanced protective effect of adding ACT to aP vaccines [3].

Erik Hewlett followed by presenting the history of ACT. A collaboration between Hewlett, Jan Wolff, and Charles Manclark led to the discovery of a 70-kDa extracytoplasmic adenylate cyclase lacking toxin activity [4], which was subsequently shown to be a fragment of the much larger ACT secreted by B. pertussis. His team demonstrated that ACT secretion and folding requires calcium, that the expression of its enzymatic activity requires calmodulin, and that ACT interacts with filamentous hemagglutinin and inhibits biofilm formation [5].

Daniel Ladant delivered a lecture in honor of Agnès Ullmann, who had passed away a few months earlier, at the age of 92 . He summarized her major contributions on $\mathrm{ACT}$, including the purification of full-length ACT, the cloning and sequencing of its structural gene $c y a A$, and the deciphering of the cya locus genetic organization and transcriptional regulation [6]. He then presented the use of ACT as antigen-delivery vehicle and provided examples of ACT-grafted antigens delivered into the cytosol of antigen-presenting cells [7]. He also illustrated the use of ACT as a tool for the study of protein-protein interactions [8]. Finally, he presented a model for ACT translocation into target cells and activation of the enzyme by calmodulin [9].

Peter Sebo illustrated the multiple and sometimes unexpected biological activities of ACT. He showed how calcium drives the formation of an intramolecular Brownian ratchet that directs unfolded ACT through the secretion system, and how ACT interacts with CD11b/CD18 on myeloid phagocytes and is then translocated. ACT blocked phagocytosis and oxidative burst, suppressed Toll-like receptor (TLR) signaling, induced apoptosis, and reprogrammed tolerogenic dendritic cells and macrophages $[10,11]$.

\section{CLINICAL AND EPIDEMIOLOGICAL ASPECTS OF PERTUSSIS}

Nicola Klein presented data on the epidemiology of pertussis in children and adolescents and noted the rapid loss of effectiveness of the reduced antigen aP vaccine in preadolescents over a 3-year period.
A human challenge model of $B$. pertussis colonization was presented by Robert Read. He described a dose-finding study and found that the dose necessary to achieve $80 \%$ colonization was $10^{5}$ colony-forming units, and that colonization peaked at day 11 and was associated with serum anti-B. pertussis antibody titer rises [12].

Helen Campbell presented an update on the national pertussis immunization program of pregnant women in England and assessed vaccine effectiveness (VE). Vaccine coverage was $72 \%$ in 2017-2018, and VE in infants $\leq 2$ months of age was $90 \%$ to $97 \%$ against fatal infant pertussis. The VE of 5-component aP vaccine was greater than that of 3-component aP vaccine. Using hospital-based surveillance in Australia, Helen Marshall reported $85 \%$ VE of maternal immunization for infants aged $\leq 2$ months and $70 \%$ VE for infants $\leq 6$ months. Among 183 infants suffering from pertussis, $16 \%$ required intensive care, $6 \%$ required assisted ventilation, and 1 infant died.

Matt Edmunds presented a retrospective South England secondary boarding school cohort study and showed that $30 \%$ had culture-confirmed, probable, or possible pertussis; $12 \%$ had asymptomatic infections; and $6 \%$ were asymptomatic carriers. The overall attack rate was $48 \%$. However, nonreporting was a problem, and he suggested that aP vaccination should be used in outbreak situations and routine adolescent aP boosters should be considered.

René Raeven compared anti-PT immunoglobulin G (IgG) values and immunoproteomic profiles of $B$. pertussis infectioninduced responses between humans with different pertussis immunization backgrounds (aP vs wP). He used multiplex immunoassays, 1- and 2-dimensional immunoblotting, and mass spectrometry, both to the usual $B$. pertussis antigens and to outer membrane vesicles (OMVs), and noted that the antibody patterns were different among the groups receiving different pertussis vaccines.

Norman Fry commented on external quality assessment for the detection of $B$. pertussis by polymerase chain reaction (PCR) in Europe. Investigators from Sweden, Finland, and England evaluated different PCR methods and compared results at 2 different times, from 28 different European laboratories. They demonstrated substantial improvement from the first to the second survey.

\section{BORDETELLA BIOLOGY AND PATHOGENESIS}

New information was provided on gene regulation in Bordetella, including new data on regulation by the 2-component systems BvgAS and RisAK [13], and by small noncoding RNA. In the virulent phase, the $B$. pertussis BvgAS system is activated, and expression of virulence-activated genes increases. In contrast, their expression decreases in the avirulent phase when B. pertussis expresses virulence-repressed genes ( $v r g s)$. The expression of these genes depends on the transcription factor RisA, 
which is phosphorylated by the histidine kinase RisK. Qing Chen investigated whether RisR, whose gene is co-transcribed with risK, may also participate in $v r g$ regulation. A risR deletion was lethal in the $\mathrm{Bvg}^{-}$phase. Under $\mathrm{Bvg}^{-}$conditions, the risR mutant grew only in the presence of suppressor mutations that resulted in a phase-locked $\mathrm{Bvg}^{+}$phenotype or mutations that reduced RisA levels. A risA/risR mutant rescued the lethality of the risR mutation. Whereas the RisA histidine kinase RisS is inactive in B. pertussis, it is intact in Bordetella bronchiseptica and functions as a phosphatase. Alterations of threonine 262 converted it to a kinase and induced a mucoid phenotype due to the expression of capsular polysaccharide genes.

Gyles Ifill generated an RNAase III active site mutant and an RNase E C-terminal deletion, and observed that half of the differentially expressed genes in the mutants were also found in the transcriptome of an $h f q$ mutant published previously [14]. Regulatory RNAs may modulate virulence, as approximately $50 \%$ of the differentially expressed genes in the RNase mutants were BvgAS regulated.

Tod Merkel showed that the baboon B. pertussis challenge model recapitulates the characteristic signs of human pertussis, including leukocytosis, paroxysmal coughing, mucus production, and B. pertussis transmission [15]. It also showed more severe disease in younger than in older animals. However, unlike in young humans, there was no significant pathology in the trachea of baboons. RisA-deficient $B$. pertussis grew well in baboons but was impaired in transmission, indicating that $\mathrm{vrgs}$ participated in B. pertussis transmission. Thibaut Naninck followed $B$. pertussis colonization of the baboon respiratory tract over time by probe-based confocal laser endomicroscopy using green fluorescent protein-expressing bacteria. He could quantify bacterial colonization and observe interactions of the bacteria with antigen-presenting cells in trachea and bronchi.

Using an infant mouse model, Karen Scanlon elucidated the role of PT in the development of severe pertussis. PT activated the pulmonary angiotensin (AT) system and inhibited the protective $\mathrm{AT}_{2}$ functions in infant mice, eventually leading to pulmonary hypertension. She also showed that angiotensin-converting enzyme inhibitors might be useful to improve disease outcome. Katharina Ernst showed that host cell cyclophilin isoforms CypA and Cyp40 are required for translocation of the PT S1 subunit into the cytosol, which might make them a target for novel therapeutic strategies against pertussis.

Françoise Jacob-Dubuisson presented studies on B. pertussis homeostasis of copper, a metal that is both essential and toxic for living organisms. Unlike for other pathogens, copper export or detoxification systems are absent or not copper-regulated in $B$. pertussis. In excess copper, only a cytoplasmic copper chaperone was induced. Under copper starvation, B. pertussis upregulated an operon coding for a putative TonB-dependent transporter and 2 proteins of unknown functions. Thus,
B. pertussis copper homeostasis is more focused on harvesting the metal than defending from its toxicity.

Iain MacArthur reported that the B. pertussis growth inhibition by hydrophobic molecules [16] is due to alterations of the Acr system, an export system of acriflavine and other small hydrophobic molecules. Mutations in acr are conserved in B. pertussis, suggesting a selective advantage for these mutations. However, neither Bordetella parapertussis nor Bordetella holmesii, also human pathogens, have these mutations. The selective advantage in becoming more sensitive to hydrophobic molecules thus remains an open question.

\section{IMMUNITY AND VACCINATION}

Kingston Mills demonstrated that B. pertussis infection induces interleukin 17 (IL-17)- and interferon gamma (IFN$\gamma)$-secreting tissue-resident memory $\mathrm{T}$ cells $\left(\mathrm{T}_{\mathrm{RM}}\right)$ in the nose and lungs of mice [17], which proliferate after reinfection, mediating rapid pathogen clearance. $\mathrm{wP}$ vaccines also primed $\mathrm{T}_{\mathrm{RM}}$, whereas aP vaccines did not and failed to protect against nasal colonization. An aP vaccine with a TLR2 agonist and c-diGMP adjuvant instead of alum induced $\mathrm{T}_{\mathrm{RM}}$ cells and opsonizing antibodies. Using PT-deficient B. pertussis strains, Cyrille Mionnet found that $\mathrm{PT}$ was required to generate mouse lung $\mathrm{CD}^{+}, \mathrm{CD}^{+}$, and $\gamma \delta \mathrm{T}_{\mathrm{RM}}$ cells. Coadministration of PT with the PT-deficient strain restored the generation of $\mathrm{T}_{\mathrm{RM}}$ cells. Adoptive transfer of $\mathrm{T}_{\mathrm{RM}}$ cells protected mice against $B$. pertussis infection.

Jeremy Ardanuy found that the type I and III interferon (IFN) receptors are important activators of inflammatory responses in B. pertussis-infected mice. Type I and III IFNs were produced in the lungs 4 days postinoculation and peaked at day 10. Infected type I IFN receptor knockout mice had lung pathology levels similar to those of wild-type mice because of increased levels of type III IFN.

Monica Cartelle Gestal identified a putative regulator, BtrS [18], implicated in the control of immunomodulation. BtrS was involved in survival within macrophages, inflammasome activation, suppression of immune cell recruitment, and persistence in the respiratory tract. Infection with a btrS mutant induced robust immunity against reinfection with all classical Bordetella.

Michel Kroes demonstrated that activation of human NK cells by $B$. pertussis is secondary to inflammasome activation and interleukin 18 secretion by macrophages. NK cells then secreted IFN- $\gamma$, which enhanced antimicrobial activity of macrophages and participated in Thl skewing.

Ricardo da Silva Antunes compared T-cell responses induced after aP boosting of adults vaccinated as infants with aP vs wP vaccines [19]. T cells remained polarized as a function of their original priming. The capacity of memory $\mathrm{CD} 4^{+} \mathrm{T}$ cells to expand after boosting was only observed in wP-primed 
individuals. Priming by either vaccine was associated with increased antibody titers and plasmablasts after boosting, suggesting that waning immunity is T-cell specific rather than B-cell specific.

Parul Kapil examined whether aP-primed Th2 responses could be polarized towards Th1 and Th17 responses in baboons after $B$. pertussis infection and found that infection of the aP-primed animals induced Th17 and Th2, but no Th1 responses. Following a second challenge with $B$. pertussis, unvaccinated convalescent animals were protected from colonization, while aP-vaccinated convalescent animals were not.

Bahaa Abu-Raya showed that maternal immunization during pregnancy interfered with the infant's antibody production. This was noted both after primary vaccination and after booster doses, and was also seen for antibody titers against diphtheria toxin and pneumococcal polysaccharides, but not for antitetanus toxin responses.

Dylan Boehm compared nasal diphtheria, tetanus, and acellular pertussis (DTaP) and DTaP plus curdlan administration in mice and found that in the absence of curdlan, DTaP accumulated in the lungs, whereas in its presence, DTaP was retained in the nasal cavity. Intranasal immunization with either vaccine decreased the bacterial burden in the lungs and induced systemic anti-PT IgG responses together with inhibition of leukocytosis after B. pertussis infection. Immunoglobulin A (IgA) was detected in nasal lavages, especially following immunization with DTaP plus curdlan.

Camille Locht explained the rationale for the live attenuated nasal pertussis vaccine BPZE1 [20]. BPZE1 protected against lung and nasal $B$. pertussis colonization in mice and baboons. Protection depended on secretory IgA and IL-17-secreting $\mathrm{T}_{\mathrm{RM}}$ cells [21]. Clinical trials in humans were performed that demonstrated safety of the vaccine and up to $100 \%$ seroconversion.

Ang Lin showed that plasmablasts and Th1-type follicular $\mathrm{T}$ lymphocytes appeared in BPZE1-vaccinated volunteers 4-14 days after vaccination, as well as BPZE1-specific Th1-polarized T cells. All BPZE1 recipients developed specific memory B cells and anti-BPZE1 IgG and IgA titers. Antigen specificity of these antibodies was broader than that of aP-vaccinated subjects and was predominantly directed to antigens not present in $\mathrm{aP}$ vaccines.

Daniela Hozbor illustrated that Bordetella OMVs [22] induced superior immunity in mice than aP vaccination. Maternal immunization did not inhibit OMV-induced immunity. Bordetella parapertussis and B. bronchiseptica OMVs protected against the respective pathogens. Protection depended on lipopolysaccharide $\mathrm{O}$ antigen and was also seen with OMVs from bacteria in the avirulent phase.

Jennifer Maynard demonstrated that humanized anti-PT monoclonal antibody hu1B7 [23] provided protection against pertussis in pertussis-challenged neonatal baboons by inhibiting intracellular PT trafficking. A modified version of hu1B7 with extended half-life was also protective, and white blood cell levels in infected baboons were inversely correlated with hu1B7 levels, which may help in defining minimum protective titers.

\section{EVOLUTIONARY AND GENOMIC ASPECTS OF BORDETELLA}

Michael Weigand presented novel approaches to analyze genome diversity including $>3500$ coding regions of $B$. pertussis. Whole-genome multiple locus sequence typing could also be directly applied on nasopharyngeal specimens.

Andrew Preston revealed that intragenomic recombination between insertion sequences caused genomic rearrangement and that different genome orders exist among B. pertussis isolates [24]. Insertion sequence-mediated recombination can result in duplication, with 12 such duplications described. They can be highly unstable $[25,26]$. Among 2430 B. pertussis isolates analyzed, 191 contained duplications, $94 \%$ of which were in 11 "hotspot" loci.

Pertussis has been treated with macrolides for decades, and Qiushui He explained that macrolide-resistant isolates are now found in China [27], as well as sporadically in Europe [28], the Middle East [29] and the United States [30]. Resistance was generally attributed to a $\mathrm{A} 2047 \mathrm{G}$ mutation in the domain $\mathrm{V}$ of the $23 \mathrm{~S} \mathrm{ri-}$ bosomal RNA gene. The frequency of this mutation increased in China and is currently at $60 \%-92 \%$.

By harnessing whole-population and age-stratified incidence data, genomic, and demographic data and vaccine coverage, Ana Bento and Pejman Rohani formulated evolutionary models of $B$. pertussis history and transmission dynamics. They concluded that there was a positive association between genetic diversity and pertussis disease incidence, suggesting that the resurgence is associated with the expansion of strains that cause more detectable symptoms.

\section{BORDETELLA OTHER THAN BORDETELLA PERTUSSIS}

Eric Harvill discussed Bordetella phylogenetics. He highlighted evidence for an environmental origin of the genus, leading to 2 independent but intersecting transmission cycles for B. bronchiseptica, 1 circulating as a respiratory pathogen in mammals and 1 as an association between the bacteria and predatory amoeba. As amoeba can colonize mammalian hosts, he speculated that B. bronchiseptica can switch between those 2 life cycles [31]. Interactions with amoeba may also represent the starting point for bacterial adaptation to eukaryotic cells. B. pertussis evolution toward a closed human-to-human lifecycle has involved genome reduction and the loss of ability to utilize amoeba as reservoir.

Tracy Nicholson characterized the B. bronchiseptica BvgR and RisAS regulons by using various isogenic mutants and measuring transcriptional responses of each mutant under 
modulating and nonmodulating growth conditions. This revealed a previously unrecognized complex regulation of virulence pathways in B. bronchiseptica.

Kacey Yount demonstrated that Bordetella colonization factor A (BcfA) [32], used as an adjuvant, enhanced immune responses to $B$. bronchiseptica and $B$. pertussis antigens. Vaccines formulated with BcfA induced protection against $B$. bronchiseptica in mice. With BcfAcontaining formulations, the Th2 responses elicited by Bordetella immunogens were attenuated, resulting in Th1/Th17 polarized responses.

\section{CONCLUSIONS}

Since the 11th International Bordetella Symposium in 2016 [33], the Bordetella research community has grown substantially, and the 12th symposium gathered more delegates than ever before.

New, up-to-date technologies were applied to further our understanding and revise some of the previous assumptions about Bordetella biology, genomics, immunology, and vaccine development. The baboon challenge model for pertussis was implemented in several laboratories and helped to refine our knowledge on $B$. pertussis infection and vaccine-induced immunity. Particularly exciting was the establishment of a controlled human challenge model for pertussis, which may be helpful to determine correlates of protection and to test novel pertussis vaccines. These advances provide hope for improved control of Bordetella infections. It will be interesting to see how these developments have evolved at the 13th International Bordetella symposium, scheduled for 2022 in Vancouver, Canada.

\section{Notes}

Financial support. The 12th International Bordetella Symposium would not have been possible without the financial support of Université Libre de Bruxelles; Université de Lille; the National Institute of Allergy and Infectious Diseases (grant number 1 R13AI 145245-01); the US Food and Drug Administration (grant number 1R13FD006613-01); Sanofi Pasteur; ILiAD Biotechnologies; GlaxoSmithKline; BioNet; and the Microbiology Society.

Potential conflicts of interest. C. L. holds patents on the BPZE1 vaccine, which is licensed to ILiAD Biotechnologies. K. H. G. M. holds a patent on TLR-2 agonists for vaccines. D. H. is a member of the Global Pertussis Initiative supported by Sanofi Pasteur, USA. K. M. E. reports grants from the Centers for Disease Control and Prevention and the National Institutes of Health; consulting fees from Merck, BioNet, and IBM; and data and safety monitoring board membership from Sanofi, X-4 Pharma, Seqirus, Moderna, and Pfizer, outside the submitted work. All other authors report no potential conflicts of interest. All authors have submitted the ICMJE Form for Disclosure of Potential Conflicts of Interest. Conflicts that the editors consider relevant to the content of the manuscript have been disclosed.

\section{References}

1. Cherry JD. Pertussis in the preantibiotic and prevaccine era, with emphasis on adult pertussis. Clin Infect Dis 1999; 28(Suppl 2):S107-11.

2. Melvin JA, Scheller EV, Miller JF, Cotter PA. Bordetella pertussis pathogenesis: current and future challenges. Nat Rev Microbiol 2014; 12:274-88.
3. Boehm DT, Hall JM, Wong TY, et al. Evaluation of adenylate cyclase toxoid antigen in acellular pertussis vaccines by using a Bordetella pertussis challenge model in mice. Infect Immun 2018; 86:e00857-17.

4. Hewlett EL, Urban MA, Manclark CR, Wolff J. Extracytoplasmic adenylate cyclase of Bordetella pertussis. Proc Natl Acad Sci U S A 1976; 73:1926-30.

5. Hoffman C, Eby J, Gray M, et al. Bordetella adenylate cyclase toxin interacts with filamentous haemagglutinin to inhibit biofilm formation in vitro. Mol Microbiol 2017; 103:214-28.

6. Glaser P, Sakamoto H, Bellalou J, Ullmann A, Danchin A. Secretion of cyclolysin, the calmodulin-sensitive adenylate cyclase-haemolysin bifunctional protein of Bordetella pertussis. EMBO J 1988; 7:3997-4004.

7. Préville X, Ladant D, Timmerman B, Leclerc C. Eradication of established tumors by vaccination with recombinant Bordetella pertussis adenylate cyclase carrying the human papillomavirus 16 E7 oncoprotein. Cancer Res 2005; 65:641-9.

8. Karimova G, Pidoux J, Ullmann A, Ladant D. A bacterial two-hybrid system based on a reconstituted signal transduction pathway. Proc Natl Acad Sci U S A 1998; 95:5752-6.

9. Voegele A, O’Brien DP, Subrini O, et al. Translocation and calmoduliun-activation of the adenylate cyclase toxin (CyaA) of Bordetella pertussis. Pathog Dis 2018; 76:fty085.

10. Cerny O, Anderson KE, Stephens LR, Hawkins PT, Sebo P. cAMP signaling of adenylate cyclase toxin blocks the oxidative burst of neutrophils through Epac-mediated inhibition of phospholipase C activity. J Immunol 2017; 198:1285-96.

11. Ahmad JN, Cerny O, Linhartova I, Masin J, Osicka R, Sebo P. cAMP signalling of Bordetella adenylate cyclase toxin through the SHP-1 phosphatase activates the BimEL-Bax pro-apoptotic cascade in phagocytes. Cell Microbiol 2016; 18:384-98.

12. De Graaf H, Ibrahim M, Hill AR, et al. Controlled human infection with Bordetella pertussis induces asymptomatic, immunizing colonization [manuscript published online ahead of print 29 September 2019]. Clin Infect Dis 2019. doi:10.1093/cid/ ciz840.

13. Chen Q, Stibitz S. The BvgASR virulence regulon of Bordetella pertussis. Curr Opin Microbiol 2019; 47:74-81.

14. Bibova I, Hot D, Keidel K, et al. Transcriptional profiling of Bordetella pertussis reveals requirement of RNA chaperone Hfq for Type III secretion system functionality. RNA Biol 2015; 12:175-85.

15. Warfel JM, Merkel TJ. The baboon model of pertussis: effective use and lessons for pertussis vaccines. Expert Rev Vaccines 2014; 13:1241-52.

16. Imaizumi A, Suzuki Y, Ono S, Sato H, Sato Y. Effect of heptakis (2,6-O-dimethyl) beta-cyclodextrin on the production of pertussis toxin by Bordetella pertussis. Infect Immun 1983; 41:1138-43.

17. Wilk MM, Misiak A, McManus RM, Allen AC, Lynch MA, Mills KHG. Lung CD4 tissue-resident memory $\mathrm{T}$ cells mediate adaptive immunity induced by previous infection of mice with Bordetella pertussis. J Immunol 2017; 199:233-43.

18. Ahuja U, Shokeen B, Cheng N, et al. Differential regulation of type III secretion and virulence genes in Bordetella pertussis and Bordetella bronchiseptica by a secreted anti- $\sigma$ factor. Proc Natl Acad Sci U S A 2016; 113:2341-8.

19. da Silva Antunes R, Babor M, Carpenter C, et al. Th1/Th17 polarization persists following whole-cell pertussis vaccination despite repeated acellular boosters. J Clin Invest 2018; 128:3853-65.

20. Locht C. Will we have new pertussis vaccines? Vaccine 2018; 36:5460-9.

21. Solans L, Debrie AS, Borkner L, et al. IL-17-dependent SIgA-mediated protection against nasal Bordetella pertussis infection by live attenuated BPZE1 vaccine. Mucosal Immunol 2018; 11:1753-62.

22. Hozbor DF. Outer membrane vesicles: an attractive candidate for pertussis vaccines. Expert Rev Vaccines 2017; 16:193-6.

23. Nguyen AW, Wagner EK, Laber JR, et al. A cocktail of humanized anti-pertussis toxin antibodies limits disease in murine and baboon models of whooping cough. Sci Transl Med 2015; 7:316ra195.

24. Weigand MR, Peng Y, Loparev V, et al. The history of Bordetella pertussis genome evolution includes structural rearrangement. J Bacteriol 2017; 199:e00806-16.

25. Caro V, Hot D, Guigon G, et al. Temporal analysis of French Bordetella pertussis isolates by comparative whole-genome hybridization. Microbes Infect 2006; 8:2228-35.

26. Dalet K, Weber C, Guillemot L, Njamkepo E, Guiso N. Characterization of adenylate cyclase-hemolysin gene duplication in a Bordetella pertussis isolate. Infect Immun 2004; 72:4874-7.

27. Wang Z, Li Y, Hou T, et al. Appearance of macrolide-resistant Bordetella pertussis strains in China. Antimicrob Agents Chemother 2013; 57:5193-4.

28. Guillot S, Descours G, Gillet Y, Etienne J, Floret D, Guiso N. Macrolide-resistant Bordetella pertussis infection in newborn girl, France. Emerg Infect Dis 2012; 18:966-8.

29. Shahcheraghi F, Nakhost Lotti M, Nikbin VS, et al. The first macrolide-resistant Bordetella pertussis strains isolated from Iranian patients. Jundishapur J Microbiol 2014; 7:1-5. 
30. Korgenski EK, Daly JA. Surveillance and detection of erythromycin resistance in Bordetella pertussis isolates recovered from a pediatric population in the Intermountain West region of the United States. J Clin Microbiol 1997; 35:2989-91.

31. Taylor-Mulneix DL, Bendor L, Linz B, et al. Bordetella bronchiseptica exploits the complex life cycle of Dictyostelium discoideum as an amplifying transmission vector. PLoS Biol 2017; 15:e2000420.
32. Jennings-Gee J, Quataert S, Ganguly T, et al. The adjuvant Bordetella colonization factor A attenuates alum-induced Th2 responses and enhances Bordetella pertussis clearance from mouse lungs. Infect Immun 2018; 86:e00935-17.

33. Carbonetti NH, Wirsing von König CH, Lan R, et al. Highlights of the 11th International Bordetella symposium: from basic biology to vaccine development. Clin Vaccine Immunol 2016; 23:842-50. 